\title{
The influence of doping sites on achieving higher thermoelectric performance for nanostructured $\alpha$-MgAgSb
}

Zihang Liu ${ }^{1,2}$, Yumei Wang ${ }^{1,3}$, Weihong Gao ${ }^{1}$, Jun $\mathrm{Mao}^{2,4}$,Huiyuan Geng ${ }^{5}$,Jing Shuai $^{2}$, Wei Cai ${ }^{1}$, Jiehe Sui ${ }^{1 *}$, and Zhifeng Ren $^{2 *}$

${ }^{1}$ National Key Laboratory for Precision Hot Processing of Metals and School of Materials Science and Engineering,Harbin Institute of Technology, Harbin 150001, China

${ }^{2}$ Department of Physics and TcSUH, University of Houston, Houston, Texas 77204, USA

${ }^{3}$ Beijing National Laboratory for Condensed Matter Physics, Institute of Physics, Chinese Academy of Sciences, Beijing 100190, China

${ }^{4}$ Department of Mechanical Engineering,University of Houston, Houston, Texas 77204, USA

${ }^{5}$ State Key Laboratory of Advanced Welding and Joining, Harbin Institute of Technology, Harbin 150001,China

Keywords:Thermoelectric materials; Doping site; Nanostructured MgAgSb; Nanotwin

* To whom correspondence should be addressed. E-mail: suijiehe@hit.edu.cn, zren@uh.edu 


\begin{abstract}
P-type nanostructured $\alpha$-MgAgSbholdsa great promise forlow temperature waste heat energy harvesting. In this work,we first investigate the effect of doping site on the carrier transport behavior of nanostructured $\alpha$-MgAgSb.Ca doping on Mg siteis rationally chosen to optimize carrier concentration and thus enhance power factor.It is demonstratedthat $\mathrm{Ca}$ dopingon the $\mathrm{Mg}$ siteleads to higher mobility and power factor in comparison withdoping on Sb site, originated from the less introduced perturbation to valence band upon doping. As the result of doping-site effect, a peak ZTof $\sim 1.3$ at $\sim 550 \mathrm{~K}$ and average ZTof $\sim 1.1$ between $300 \mathrm{~K}$ and 550 Kare achieved by Ca doping on the Mg site. Our current work on only points out that doping on the site that has less influence on the charge-conducting band should be a critical doping principle in thermoelectric materials field, but also highlights the promising prospect of nanostructured $\alpha$-MgAgSb for low temperature power generation.
\end{abstract}

\title{
1. Introduction
}

Thermoelectric materials have received extensive interests for clean energy harvesting[1-5].However, this promising prospect is hampered by the relatively low conversionefficiencyof thermoelectric devices, which is primarily determined by the materials' thermoelectricfigure of merit, $Z T=S^{2} \sigma \mathrm{T} /\left(\kappa_{\text {lat }}+\kappa_{\text {ele }}\right)$, where $S, \sigma, T, \kappa_{\text {lat }}$, and $\kappa_{\text {ele }}$ are the Seebeck coefficient, electrical conductivity, absolute temperature, lattice thermal conductivity,andelectronic thermal conductivity, respectively.Due to the inherent trade-off between $\sigma$ and Sviacarrier concentration[1],tuning carrier concentration via effective doping has long been regarded as the most straightforward approach to enhance power factor $\left(P F=\sigma S^{2}\right)$ for achieving high output power density[6-9], especially for heavy band systemthat needs a high carrier concentration to reach[10, 11].Generally different dopants at different lattice sites are considered equivalent ifacomparable carrier concentration is finally achieved in the rigid band approximation[1, 6, 8], becausethe corresponding ionized impurity scattering for 
carriers merely depends on dielectric constant,doping concentration and carrier concentration for a specific compound[12, 13].In fact, valence band is normally dominated byanion atomic orbitals while conduction band by cation orbitals regarding an ionic lattice, first predicted by Ioffe[14]. Therefore, dopingon anion or cation sites would introduce the perturbation to valence or conduction band that may affect the corresponding carrier mobility of hole or electron [14], respectively. However, the dopingeffect on which site has long been disregarded even though it may have a significant influence on the carrier transport behavior in heavily doped semiconductors.For instance, n-type $\mathrm{PbTe}_{1-\mathrm{x}} \mathrm{Se}_{\mathrm{x}}$ compounds have a higher mobility than thep-type counterparts owing to the corresponding perturbation to valence band with Se alloying on Te site[14].

For low temperature power generation applications,p-type BiSbTe alloy hasalways been the only promising materialsince 1950s[16-19].Recently, mechanically robustnanostructured $\alpha$-MgAgSbbased alloys with superior thermoelectric performance was successfully synthesized by a simple two-steps ball milling and quick hot press method[20-27]. $\alpha$-MgAgSb has only one heavy valence band located at the X-pointin the Brillouin zone[28-30],which requireshigh carrier concentration to achieve good thermoelectric properties [25].Thus, previous workmainly focused on tuning carrier concentration by doping on the Mgor Ag site[20-22, 25]. So far there is no relevant report about the dopingon the Sb site in the nanostructured $\alpha$-MgAgSb, which offers a chance to compare the role of doping atcation and anionsites on the carrier transport behavior.

Herein, Ca doping on the $\mathrm{Mg}$ sitewas first chosen to optimize the carrier concentration and thus enhance the power factor. Then, we studied the electrical transport properties of doping on the Sb site, including As, Bi, Sn and Pb.Compared with $\mathrm{Ca}$ and $\mathrm{Li}$ doping on the $\mathrm{Mg}$ site, it can be clearly observed that doping on $\mathrm{Sb}$ site gave riseto much lower mobility and power factor due to the influence on the valence band. Finally, we have achieved a peak ZTof $\sim 1.3$ at $~ 550 \mathrm{~K}$ and an average ZTof $\sim 1.1$ between $300 \mathrm{~K}$ and $550 \mathrm{~K}$ upon Ca doping on the Mg site.Our work sheds 
new light on the carrier transport behavior of nanostructureda-MgAgSb, which could help and contribute to realizing the rational doping in other thermoelectric systems.

\section{Experimental section}

Synthesis.All the chemicals were purchased from Alfa Aesar and then weighed according to the nominal composition $\mathrm{Mg}_{1-\mathrm{x}} \mathrm{Ca}_{\mathrm{x}} \mathrm{Ag}_{0.97} \mathrm{Sb}_{0.99}$ samples $(\mathrm{x}=0,0.0025$, 0.005 , and 0.01 ). The synthesis processwas totally identical with our previous reports [20-25].

X-ray diffraction. X-ray diffraction (XRD) analysis was performed using a PANa-lytical multipurpose diffractometer with an X'celerator detector (PANalyticalX'Pert Pro). The phases were analyzed with JADE 6.0 software. The microstructure was observed by a scanning electron microscope (SEM, JEOL 6330F) and a high resolution transmission electron microscope (HRTEM, JEOL 2100F). Thin TEM specimens were prepared by conventional methods, including cutting, grinding, dimpling, and Ar-ion milling from the bottom and top surfaces of the discs with a liquid nitrogen cooling stage operated at $2.5 \mathrm{keV}$ and an incidence angle of $3^{\circ}$ using a Gatan PIPS 695. Finally, Ar-ion milling with lower-voltage and incidence angle was continually performed for 1 hour to clean out the amorphous layer on the surface.

Physical Characterization.Bar samples were cut from the disks and used for simultaneous measurement of the electrical resistivity $(\rho)$ and Seebeck coefficient $(S)$ on a commercial system (ULVAC ZEM-3). The thermal conductivity was calculated using $\kappa=D C_{p} d$, where $D, C_{p}$, and $d$ are the thermal diffusivity, specific heat capacity, and density, respectively. The thermal diffusivity coefficient $(D)$ was measured using the coin sample on a laser flash system (Netzsch LFA 457, Germany). The specific heat capacity $\left(C_{p}\right)$ was measured on a differential scanningcalorimetry thermal analyzer (Netzsch DSC 404 C, Germany), shown in the Figure S1. The density (d) around $6.2 \mathrm{~g} \mathrm{~cm}^{-3}$ was determined by the Archimedes method. The Hall Coefficient $R_{H}$ at room temperature was measured using the PPMS (Physical Properties 
Measurement System, Quantum Design). The Hall carrier concentration $\left(n_{H}\right)$ was obtained by $n_{H}=1 / e R_{H}$ and the Hall carrier mobility $\left(\mu_{\mathrm{H}}\right)$ was calculated by $\sigma=e \mu_{H} n_{H}$, where $e$ is the electronic charge and $\sigma$ the electrical conductivity.

Theoretical calculation.The electronic structure of $\alpha$-MgAgSb was calculated by the density-functional theory (DFT) as implemented in the open-source Quantum Espresso program package[31]. We used the projected augment wave (PAW) potentials, provided by the standard solid-state pseudo potential library. The exchange-correlation function was taken within the generalized gradient approximation (GGA) in the parameterization of Perdew-Burke-Ernzerhof (PBE)[32]. A plane-wave basis was adopted for the expansion of the valence electron wavefunctions with kinetic-energy cutoffs of 50 Ry. For ground state calculations we used an $8 \times 8 \times 8$ Monkhorst-Pack k-point mesh.In addition, the spin-orbit couplings were not included in the current calculations.

\section{Results}

Figure 1a shows the X-ray diffraction (XRD) patterns of $\mathrm{Mg}_{1-\mathrm{x}} \mathrm{Ca}_{\mathrm{x}} \mathrm{Ag}_{0.97} \mathrm{Sb}_{0.99}$ samples $(x=0,0.0025,0.005$, and 0.01).The diffraction peaks of all the samples exhibit goodmatchwith the $\alpha-\mathrm{MgAgSb}$ (space group $I \overline{4} C 2$ ) and no impurity phases could be observed within the detection limit of the XRD spectrometer[33].Besides, the XRD peaks shift to low angle with increasing Ca alloying content (Figure 1b), which implies that Ca may totally enter into Mg site in the MgAgSb lattice.

Figure 2a reveals that the typical grain size, ranging from 100 to $200 \mathrm{~nm}$, is significantly smaller, compared with that of the report by Ying et al[28]. The ubiquitous in-situ nanoinclusions around $10 \mathrm{~nm}$ can be observed in Figure 2b, similar to the nanostructured BiSbTe[17].Those plentiful grain boundaries and interfaces with different orientation and atomicspacing increase the phonons scattering with medium-to-long mean free path[34, 35].

Besides nanograinsandnanoinclusions, two types of nanotwins, lamellar and 
spearhead shaped, can be clearly observed in Figure 3a and 3b, respectively. The typical width of nanotwinsis on the order of several nanometers, much smaller than that in nanotwinned copper fabricated by pulsed electrodeposition[36]. The inset in Figure 3a presents the corresponding fast Fourier transformation (FFT) pattern along the [011] zone axis direction, which indicates a typical type-I twin relationship $(41 \overline{1})_{\mathrm{M}}$ and $(41 \overline{1})_{\mathrm{T}}$ among the lamellae that are symmetrical to each other with respect to the (233) plane.The presence of the tapering twins is generally as a consequence of decreasing boundaryarea and thus lowering the boundaryenergy[37]. Those cleanandcoherent nanotwin boundaries act as additional phonon scattering centerswithout degrading the electrical transport properties[34]. Furthermore, the nanotwin boundaries could be able toselectively scatterlow-energycarriersbypotentialbarrierscattering, therefore enhancing the Seebeckcoefficient or suppressing the bipolar effect[2, 38]. That is why nanostructured MgAgSb has a comparable electrical resistivity but a higher Seebeck coefficient in comparison with coarse MgAgSb[28]. The mechanism of nanotwin formation in nanostructured MgAgSbmay be related to the high energy ball milling process that normally generatessubstantially higher angle micro and nano-sized grain boundaries as well as deformation and stress[39]. Since therequired formationenergy for twin boundary is muchlower than the conventional high angle grain boundary, the presence of twin will thus reducethe total interfacial energy under a thermodynamic situation[37]. In addition, compounds with phase transformation behavior prefer to form the twin structure, as observed in a variety ofalloys[40, 41].

Figure 4 presents the temperature dependent thermoelectric properties of $\mathrm{Mg}_{1-\mathrm{x}} \mathrm{Ca}_{\mathrm{x}} \mathrm{Ag}_{0.97} \mathrm{Sb}_{0.99}$ samples. Electrical resistivity $\rho$ monotonically decreaseswith increasing Ca doping concentration at the whole measured temperature range (Figure 4a). Specifically, room-temperature $\rho$ of $\mathrm{Mg}_{0.99} \mathrm{Ca}_{0.01} \mathrm{Ag}_{0.97} \mathrm{Sb}_{0.99}\left(\sim 1.8 \times 10^{-5} \Omega \mathrm{m}\right)$ is about half of that of the undoped $\operatorname{MgAg}_{0.97} \mathrm{Sb}_{0.99}\left(\sim 3.6 \times 10^{-5} \Omega \mathrm{m}\right)$. Since $\mathrm{CaAgSb}$ compound exhibits a typical semi-metal behavior and its room-temperature $\rho$ is around 
$2.5 \times 10^{-6} \Omega \mathrm{m}[42], \rho$ of $\mathrm{Ca}$ doped MgAgSb samples will be lower than pristine MgAgSb when Ca alloying on the Mg site. Due to the increased carrier concentrationshown in Table 1,Sdecreases somewhat upon doping (Figure 4b), in accordance with the tendency of $\rho$. In addition, MgAgSb based materials exhibit peak values of Seven below 400Kowing to the very small band gap or semi-metal characteristic[26, 28-30,43].This is related with the onset of bipolar effect, because minority carriers, namely electron in p-type MgAgSb, are thermally excitedacross the band gap and lead to the reducedS.Power factor $\left(P F=S^{2} \sigma\right)$ calculated from the measured electrical resistivity and Seebeck coefficient is plotted in Figure 4c.Compared with the pristine $\mathrm{MgAg}_{0.97} \mathrm{Sb}_{0.99}$, PFisenhancedaround 20\% upon Ca doping due to the optimized carrier concentration. Figure $4 \mathrm{~d}$ showsthe total thermal conductivity $\kappa_{\text {total }}$ first decreases slightly because of Umklapp scattering and then increases significantly due to the bipolar effectat higher temperature.Since electronic thermal conductivity $\kappa_{\text {ele }}$ can be calculated from theWiedemann-Franz relationship, $\kappa_{\text {ele }}$ $=L \sigma T$, where $L$, Lorenz number, is calculated assuming the single parabolic band modelbased on Equation (1-4)[44, 45]:

$$
\begin{gathered}
S=\frac{k_{B}}{e}\left(\frac{(r+5 / 2) F_{(r+3 / 2)}(\eta)}{(r+3 / 2) F_{(r+1 / 2)}(\eta)}-\eta\right) \\
F_{n}(\eta)=\int_{0}^{\infty} \frac{\chi^{\eta}}{1+e^{\chi-\eta}} d \chi \\
\eta=\frac{E_{f}}{k_{B} T} \\
L=\left(\frac{k_{B}}{e}\right)^{2}\left\{\frac{(r+7 / 2) F_{(r+5 / 2)}(\eta)}{(r+3 / 2) F_{(r+3 / 2)}(\eta)}-\left[\frac{(r+5 / 2) F_{(r+3 / 2)}(\eta)}{(r+3 / 2) F_{(r+1 / 2)}(\eta)}\right]^{2}\right\}
\end{gathered}
$$

where $\mathrm{k}_{\mathrm{B}}$ is the Boltzmann constant, e is the electron charge, $\eta$ is the reduced Fermi energy, $F_{n}(\eta)$ is the $n^{\text {th }}$ order Fermi integral, $r$ is the scattering factor.As acoustic phonon scattering is the main scattering mechanism for most thermoelectric materials above room temperature[1, 6],the scattering factor $r$ could be considered as $-1 / 2$ in 
calculation.Lattice thermal conductivity $\kappa_{\text {lat }}$ is obtained by subtracting $\kappa_{\text {ele }}$ from $\kappa_{\text {total }}$ before intrinsic excitation, shown in Figure 4e. Apparently, Ca doping leads to the gradual suppressionof low-temperature $\kappa_{\text {lat }}$, whichis related with the corresponding point defect scattering between host atom (Mg) and alloying atom (Ca)because of mass fluctuation and surrounding strain field fluctuation. Due to thelarger increase inPFthan the smaller increase in total thermal conductivity, ZT has been improved at the whole measured temperature rangeupon Ca doping(Figure 4f). The highest peak $Z T$ and average $Z T$, obtained inMg${ }_{0.995} \mathrm{Ca}_{0.005} \mathrm{Ag}_{0.97} \mathrm{Sb}_{0.99}$,reaches $\sim 1.3$ and $\sim 1.1$, respectively, even higher than those of $\mathrm{Mg}_{0.99} \mathrm{Li}_{0.01} \mathrm{Ag}_{0.97} \mathrm{Sb}_{0.99}(\sim 1.2, \sim 1.0)$ [25].

Figure 5 shows the temperature dependent electrical transport properties for $\operatorname{MgAg}_{0.97} \mathrm{Sb}_{0.99-\mathrm{x}} \mathrm{M}_{\mathrm{x}}(\mathrm{M}=\mathrm{As}, \mathrm{Bi}, \mathrm{Sn}$ and $\mathrm{Pb})(\mathrm{x}=0,0.005$ and 0.01). Table 2 shows their corresponding carrier concentration and mobility. As shown in the Figure 5a, with increasing doping concentration, As doping obviously decreases the $\rho$ while $\mathrm{Bi}$ doping slightly increases the $\rho$. This distinct behavior may be related with the change of band gap or the difference of electronegativity upon isovalent doping [46-49],which need further experiments and theoretical calculations to support this speculation that is beyond our scope of present work. As expected, As doping leads to the substantial reduction of Sbecause of the increased carrier concentration (Figure 5b). In contrast, Bi doping does not change $S$ too much (Figure 5b). Finally, $P F$ increases moderatelyupon As doping, but Bi doping lowers the $P F$ (Figure 5c).

Due to the acceptor characteristic of $\mathrm{Sn}$ or $\mathrm{Pb}, \rho$ decreases somewhat shown in the Figure $5 \mathrm{~d}$. It should be mentioned that Sn doping leads to a much lower $\rho$ compared with that of $\mathrm{Pb}$ doping. The relatively high doping effectiveness of $\mathrm{Sn}$ doping is possibly ascribed to the very small difference of atom mass and radius between $S n$ and Sb. The increased carrier concentration leads to the decreased $S$ upon Sn or Pb doping (Figure 5e). As a result, Figure $5 f$ shows that $P F$ of doped samples are almost same with pristine sample although the highest carrier concentration of Sn doping could reach $5.0 \times 10^{19} \mathrm{~cm}^{-3}$. Given the above results, it is quite clear that doping on $\mathrm{Sb}$ site gives rise to the lower $P F$ despite the increased carrier 
concentration, compared with that of Ca doping on Mg site.

\section{Discussion}

Figure 6 shows the calculatedpartial density of state (DOS) of $\alpha$-MgAgSb. The calculated band structure andtotal DOS have been given somewhere else[27]. It is obvious that $\mathrm{p}$ orbital of Sb exhibits a significantly higher DOS than other atom orbital near the Fermi level, consistent with the recent reports[28, 43, 50].This indicates that the high total DOS of heavy valence bandis predominantlydecided by the p orbital of Sb. In general, valence band is dominated byanion atomic orbitals while conduction band by cation orbitals regarding an ionic lattice[14], in perfect accordance with the present calculation results.

Historically, different dopants at different sites are considered equivalent when reaching the same carrier concentration in the rigid band approximation. However, it may have a significant influence on the carrier transport behavior due to the perturbation to the corresponding band [14].For example,anion dopants normally lead to higher mobility than cation dopants at high carrier concentration in n-type PbSesystem[15].In order to investigate this often-overlooked effect, taking MgAgSb as a sample, we compare the electrical transport properties of $\mathrm{Ca}$ doping and $\mathrm{Li}$ doping at Mg sitewith dopants on the Sb site, including As, Bi, Sn and Pb (Figure 7).

The dashed linesin the Figure7 are calculated based on Equations (1-3, 5, 6) assuming the single parabolic band model [44].

$$
\begin{aligned}
& n_{H}=\frac{\left(2 m^{*} k_{B} T\right)^{3 / 2}}{3 \pi^{2} \mathrm{~h}^{3}} \frac{(r+3 / 2)^{2} F_{(r+1 / 2)}^{2}(\eta)}{(2 r+3 / 2) F_{(2 r+1 / 2)}(\eta)} \\
& \mu_{H}=\left[\frac{e \pi \mathrm{h}^{4}}{\sqrt{2}\left(k_{B} T\right)^{3 / 2}} \frac{d v_{L}^{2}}{E_{d e f}^{2}\left(m^{*}\right)^{5 / 2}} \frac{(2 r+3 / 2) F_{(2 r+1 / 2)}(\eta)}{(r+3 / 2)^{2} F_{(r+1 / 2)}(\eta)}\right]
\end{aligned}
$$

wherem ${ }^{*}$ is the total density of state (DOS)effective mass, dis density $\sim 6.2 \mathrm{gcm}^{-3}, v_{L}$ is the longitudinalvelocity of sound $\sim 3708 \mathrm{~m} \mathrm{~s}^{-1}$ [28], $E_{\text {def }}$ is deformationpotential coefficient $6.3 \mathrm{eV}$ [24].As shown in Figure 7a, the mobility reduction of doping on 
Mg site matches well withthe theoretical model.However, dopants on Sbsite lead to a lower carrier mobility at the same carrier concentration. As known, acoustic phonon scattering is generally assumed to be the dominating origin for carrier transport in semiconductor, whose strength is decided by the intrinsic material parameters such as material density, longitudinal velocity ofsound, effective mass, anddeformationpotential coefficient[6, 51].Given that the present doping concentration would not result in a substantialinfluence on those parameters, additional carrier scattering center must be considered for the mobilitydifference. Ionized impurity scatteringmerely depends on dielectric constant,doping concentration and carrier concentration for a specific compound, which is typically independent of doping-site. Because doping on the Sb site leads to a reasonable change of carrier concentration, dopants may totally enter into the Sb site considering the present ultralow doping concentration. Therefore, the only remaining factor isthe introduced perturbation to the valence or conduction band upon doping,as previously discussed.Since high total DOS of heavy valence band is predominantlydecided by the p orbital of Sb, doping on the Sb site would introduce the perturbation to the valence band while doping on the $\mathrm{Mg}$ site have less extra influence on the valence band. Thus, doping on Sb site maydeteriorate the corresponding carrier mobility, eventually much lower than doping on the Mg site.

Due to the different mobility upon doping, Figure 7bshows thatlower $\rho$ can beobtained by rational doping of $\mathrm{Ca}$ on the $\mathrm{Mg}$ site. Figure 7c shows that all the experimental data fall nearly on the calculated Pisarenko plotwith $m^{*}=2.4 m_{e}$, which clearly illustratesthat band structure is almost unchanged andirrelevant with doping-site.Finally, carrier concentrationdependent $P F$ of doping on the $\mathrm{Mg}$ site is basically consistent with the theoretical model, which is obviously higher than that of doping on the Sb site, especially in the heavily doped range (Figure7d). Therefore, in order to achieve optimum mobility and $P F$, doping on the right site that has less influence on the charge-conducting bandshould be acritical doping principle. 


\section{Conclusion}

In summary, we compare the electrical transport properties of dopants on different sites in nanostructured $\alpha$-MgAgSb. Thenoticeably lower carrier mobility and power factor of doping on Sb site is due to the introduced perturbation to valence band. In contrast, power factor could be enhanced around 20\% via Ca doping on Mg site that is basically consistent with the theoretical model. As a result, a peak ZTof $\sim 1.3$ at $550 \mathrm{~K}$ and average ZTof $\sim 1.1$ between $300 \mathrm{~K}$ and $550 \mathrm{~K}$ are realized byrationally choosing Ca doping.Our work not only elucidates the critical role of doping site on the electrical transport behavior of nanostructured $\alpha$-MgAgSb, but also may be applicable to other thermoelectric systems and help optimize the corresponding properties.

\section{Acknowledgement}

The work performed at the University of Houston was funded by the US Department of Energy under Contract Number DOE DE-SC0010831 (sample preparation and characterizations); this work was also supported byNational Natural ScienceFoundation of China (no. 51622101, 51471061 and 51271069) (Calculation part). 


\section{Reference}

[1] G.J. Snyder, E.S. Toberer, Complex thermoelectric materials, Nature Materials 7(2) (2008) 105-114.

[2] A. Minnich, M. Dresselhaus, Z. Ren, G. Chen, Bulk nanostructured thermoelectric materials: current research and future prospects, Energy \& Environmental Science 2(5) (2009) 466-479.

[3] J. Yang, T. Caillat, Thermoelectric materials for space and automotive power generation, MRS bulletin 31(03) (2006) 224-229.

[4] X. Shi, L. Chen, C. Uher, Recent advances in high-performance bulk thermoelectric materials, International Materials Reviews 61 (06) (2016) 379-415.

[5] G. Tan, L.-D. Zhao, M.G. Kanatzidis, Rationally Designing High-Performance Bulk Thermoelectric Materials, Chemical Reviews 116(19) (2016) 12123-12149.

[6] H.J. Goldsmid, Introduction to thermoelectricity, Springer Science \& Business Media2009.

[7] Y. Pei, A. LaLonde, S. Iwanaga, G.J. Snyder, High thermoelectric figure of merit in heavy hole dominated PbTe, Energy \& Environmental Science 4(6) (2011) 2085-2089.

[8] Y. Pei, H. Wang, G.J. Snyder, Band engineering of thermoelectric materials, Advanced Materials 24(46) (2012) 6125-6135.

[9] W. Liu, Q. Jie, H.S. Kim, Z. Ren, Current progress and future challenges in thermoelectric power generation: From materials to devices, Acta Materialia 87 (2015) 357-376.

[10] X. Shi, J. Yang, J.R. Salvador, M. Chi, J.Y. Cho, H. Wang, S. Bai, J. Yang, W. Zhang, L. Chen, Multiple-filled skutterudites: high thermoelectric figure of merit through separately optimizing electrical and thermal transports, Journal of the American Chemical Society 133(20) (2011) 7837-7846.

[11] J. Li, J. Sui, Y. Pei, C. Barreteau, D. Berardan, N. Dragoe, W. Cai, J. He, L.-D. Zhao, A high thermoelectric figure of merit ZT> 1 in Ba heavily doped BiCuSeO oxyselenides, Energy \& Environmental Science 5(9) (2012) 8543-8547.

[12] P. Debye, E. Conwell, Electrical properties of N-type germanium, Physical Review 93(4) (1954) 693.

[13] S. Wang, J. Yang, L. Wu, P. Wei, W. Zhang, J. Yang, On Intensifying Carrier Impurity Scattering to Enhance Thermoelectric Performance in Cr-Doped $\mathrm{Ce}_{\mathrm{y}} \mathrm{Co}_{4} \mathrm{Sb}_{12}$, Advanced Functional Materials 25(42) (2015) 6660-6670.

[14] D. Khokhlov, Lead chalcogenides: physics and applications, CRC Press2002.

[15] H. Goldsmid, R. Douglas, The use of semiconductors in thermoelectric refrigeration, British Journal of Applied Physics 5(11) (1954) 386.

[16] B. Poudel, Q. Hao, Y. Ma, Y. Lan, A. Minnich, B. Yu, X. Yan, D. Wang, A. Muto, D. VashaeeX. Chen, J. Liu, M. S. Dresselhaus, G. Chen, Z. Ren, High-thermoelectric performance of nanostructured bismuth antimony telluride 
bulk alloys, Science 320(5876) (2008) 634-638.

[17] J. Li, Q. Tan, J.F. Li, D.W. Liu, F. Li, Z.Y. Li, M. Zou, K. Wang, BiSbTe-Based Nanocomposites with High ZT : The Effect of SiC Nanodispersion on Thermoelectric Properties, Advanced Functional Materials 23(35) (2013) 4317-4323.

[18] F. Hao, P. Qiu, Y. Tang, S. Bai, T. Xin, H. Chu, Q. Zhang, P. Lu, T. Zhang, D. Ren, J. Chen, X. Shi and L. Chen, High efficiency $\mathrm{Bi}_{2} \mathrm{Te}_{3}$-based materials and devices for thermoelectric power generation between 100 and $300^{\circ} \mathrm{C}$, Energy \& Environmental Science 9 (2016) 3120-3127

[19] H. Zhao, J. Sui, Z. Tang, Y. Lan, Q. Jie, D. Kraemer, K. McEnaney, A. Guloy, G. Chen, Z. Ren, High thermoelectric performance of MgAgSb-based materials, Nano Energy 7 (2014) 97-103.

[20] J. Shuai, H.S. Kim, Y. Lan, S. Chen, Y. Liu, H. Zhao, J. Sui, Z. Ren, Study on thermoelectric performance by $\mathrm{Na}$ doping in nanostructured $\mathrm{Mg}_{1-\mathrm{x}} \mathrm{Na}_{\mathrm{x}} \mathrm{Ag}_{0.97}$ $\mathrm{Sb}_{0.99}$, Nano Energy 11 (2015) 640-646.

[21] J. Sui, J. Shuai, Y. Lan, Y. Liu, R. He, D. Wang, Q. Jie, Z. Ren, Effect of Cu concentration on thermoelectric properties of nanostructured p-type $\mathrm{MgAg}_{0.97-}$ ${ }_{\mathrm{x}} \mathrm{Cu}_{\mathrm{x}} \mathrm{Sb}_{0.99}$, Acta Materialia 87 (2015) 266-272.

[22] D. Kraemer, J. Sui, K. McEnaney, H. Zhao, Q. Jie, Z. Ren, G. Chen, High thermoelectric conversion efficiency of MgAgSb-based material with hot-pressed contacts, Energy \& Environmental Science 8(4) (2015) 1299-1308.

[23] Z. Liu, J. Shuai, J. Mao, Y. Wang, Z. Wang, W. Cai, J. Sui, Z. Ren, Effects of antimony content in $\mathrm{MgAg}_{0.97} \mathrm{Sb}_{\mathrm{x}}$ on output power and energy conversion efficiency, Acta Materialia 102 (2016) 17-23.

[24] Z. Liu, Y. Wang, J. Mao, H. Geng, J. Shuai, Y. Wang, R. He, W. Cai, J. Sui, Z. Ren, Lithium Doping to Enhance Thermoelectric Performance of MgAgSb with Weak Electron-Phonon Coupling, Advanced Energy Materials 6(7) (2016) 1502269.

[25] Z. Liu, W. Gao, X. Meng, X. Li, J. Mao, Y. Wang, J. Shuai, W. Cai, Z. Ren, J. Sui, Mechanical properties of nanostructured thermoelectric materials $\alpha-\mathrm{MgAgSb}$, Scripta Materialia 127 (2017) 72-75.

[26] Z. Liu, H. Geng, J. Mao, S. Jing, R. He, C. Wang, W. Cai, J. Sui, Z. Ren, Understanding and manipulating the intrinsic point defect in $\alpha$-MgAgSb for higher thermoelectric performance, Journal of Materials Chemistry A (2016)DOI: 10.1039/C6TA06832D.

[27] P. Ying, X. Liu, C. Fu, X. Yue, H. Xie, X. Zhao, W. Zhang, T. Zhu, High Performance $\alpha$-MgAgSb Thermoelectric Materials for Low Temperature Power Generation, Chemistry of Materials 27(3) (2015) 909-913.

[28] D. Li, H. Zhao, S. Li, B. Wei, J. Shuai, C. Shi, X. Xi, P. Sun, S. Meng, L. Gu, Atomic Disorders Induced by Silver and Magnesium Ion Migrations Favor High Thermoelectric Performance in $\alpha$-MgAgSb-Based Materials, Advanced Functional Materials 25(41) (2015) 6478-6488. 
[29] C. Sheng, H. Liu, D. Fan, L. Cheng, J. Zhang, J. Wei, J. Liang, P. Jiang, J. Shi, Predicting the optimized thermoelectric performance of MgAgSb, Journal of Applied Physics 119(19) (2016) 195101.

[30] P. Giannozzi, Quantum ESPRESSO: a modular and open-source software project for quantum simulations of materials, Journal of Physics Condensed Matter 21(39) (2009) 395502-395520(19).

[31] J.P. Perdew, K. Burke, M. Ernzerhof, Generalized Gradient Approximation Made Simple, Physical Review Letters 77 (1996)3865

[32] M.J. Kirkham, A.M. dos Santos, C.J. Rawn, E. Lara-Curzio, J.W. Sharp, A.J. Thompson, Abinitio determination of crystal structures of the thermoelectric material MgAgSb, Physical Review B 85(14) (2012) 144120.

[33] D. Medlin, G. Snyder, Interfaces in bulk thermoelectric materials: a review for current opinion in colloid and interface science, Current Opinion in Colloid \& Interface Science 14(4) (2009) 226-235.

[34] Y. Lan, A.J. Minnich, G. Chen, Z. Ren, Enhancement of Thermoelectric Figure-of-Merit by a Bulk Nanostructuring Approach, Advanced Functional Materials 20(3) (2010) 357-376.

[35] L. Lu, Y. Shen, X. Chen, L. Qian, K. Lu, Ultrahigh strength and high electrical conductivity in copper, Science 304(5669) (2004) 422-426.

[36] J. Zhang, Y. Zheng, L. Zhao, The structure and mobility of the intervariant boundaries in 18R martensite in a $\mathrm{Cu}-\mathrm{Zn}-\mathrm{Al}$ alloy, Acta Materialia 47(7) (1999) 2125-2141.

[37] J. Mao, Y. Wang, H.S. Kim, Z. Liu, U. Saparamadu, F. Tian, K. Dahal, J. Sun, S. Chen, W. Liu, High thermoelectric power factor in $\mathrm{Cu}-\mathrm{Ni}$ alloy originate from potential barrier scattering of twin boundaries, Nano Energy 17 (2015) 279-289.

[38] D. Zhang, Processing of advanced materials using high-energy mechanical milling, Progress in Materials Science 49(3) (2004) 537-560.

[39] K. Otsuka, X. Ren, Physical metallurgy of Ti-Ni-based shape memory alloys, Progress in Materials Science 50(5) (2005) 511-678.

[40] D. Wu, L.-D. Zhao, S. Hao, Q. Jiang, F. Zheng, J.W. Doak, H. Wu, H. Chi, Y. Gelbstein, C. Uher, C. Wolverton, M. Kanatzidis, J. He, Origin of the high performance in GeTe-based thermoelectric materials upon $\mathrm{Bi}_{2} \mathrm{Te}_{3}$ doping, Journal of the American Chemical Society 136(32) (2014) 11412-11419.

[41] J. Wang, X.-C. Liu, S.-Q. Xia, X.-T. Tao, $\mathrm{Ca}_{1-\mathrm{x}} \mathrm{RE}_{\mathrm{x}} \mathrm{Ag}_{1-\mathrm{y}} \mathrm{Sb}(\mathrm{RE}=\mathrm{La}, \mathrm{Ce}, \mathrm{Pr}, \mathrm{Nd}$, Sm; $0 \leq \mathrm{x} \leq 1 ; 0 \leq \mathrm{y} \leq 1)$ : Interesting Structural Transformation and Enhanced High-Temperature Thermoelectric Performance, Journal of the American Chemical Society 135(32) (2013) 11840-11848.

[42] N. Miao, P. Ghosez, Optimization of Thermoelectric Properties of MgAgSb-Based Materials: A First-Principles Investigation, The Journal of Physical Chemistry C 119(25) (2015) 14017-14022.

[43] J. Wang, X.-C. Liu, S.-Q. Xia, X.-T. Tao, $\mathrm{Ca}_{1-x} \mathrm{RE}_{\mathrm{X}} \mathrm{Ag}_{1-\mathrm{y}} \mathrm{Sb}(\mathrm{RE}=\mathrm{La}, \mathrm{Ce}, \mathrm{Pr}, \mathrm{Nd}$, 
Sm; $0 \leq \mathrm{x} \leq 1 ; 0 \leq \mathrm{y} \leq 1)$ : Interesting Structural Transformation and Enhanced High-Temperature Thermoelectric Performance, Journal of the American Chemical Society 135(32) (2013) 11840-11848.

[44] A.F. May, E.S. Toberer, A. Saramat, G.J. Snyder, Characterization and analysis of thermoelectric transport in n-type $\mathrm{Ba}_{8} \mathrm{Ga}_{16-x} \mathrm{Ge}_{30+x}$, Physical Review B 80(12) (2009) 125205.

[45] J. Shen, Z. Chen, L. Zheng, W. Li, Y. Pei, Single parabolic band behavior of thermoelectric p-type CuGaTe 2 , Journal of Materials Chemistry C 4(1) (2016) 209-214.

[46] E.S. Toberer, C.A. Cox, S.R. Brown, T. Ikeda, A.F. May, S.M. Kauzlarich, G.J. Snyder, Traversing the Metal-Insulator Transition in a Zintl Phase: Rational Enhancement of Thermoelectric Efficiency in $\mathrm{Yb}_{14} \mathrm{Mn}_{1-\mathrm{x}} \mathrm{Al}_{\mathrm{x}} \mathrm{Sb}_{11}$, Advanced Functional Materials 18(18) (2008) 2795-2800.

[47] J.H. Lee, J. Wu, J.C. Grossman, Enhancing the Thermoelectric Power Factor with Highly Mismatched Isoelectronic Doping, Physical Review Letters 104(1) (2010) 016602.

[48] L.D. Zhao, H.J. Wu, S.Q. Hao, C.I. Wu, X.Y. Zhou, K. Biswas, J.Q. He, T.P. Hogan, C. Uher, C. Wolverton, V. P. Dravid, M. G. Kanatzidis, All-scale hierarchical thermoelectrics: MgTe in PbTe facilitates valence band convergence and suppresses bipolar thermal transport for high performance, Energy \& Environmental Science 6(11) (2013) 3346-3355.

[49] N. Miao, J. Zhou, B. Sa, B. Xu, Z. Sun, Pressure-induced semimetal-semiconductor transition and enhancement of thermoelectric performance in $\alpha$-MgAgSb, Applied Physics Letters 108(21) (2016) 213902.

[50] H. Wang, X. Cao, Y. Takagiwa, G.J. Snyder, Higher mobility in bulk semiconductors by separating the dopants from the charge-conducting band - a case study of thermoelectric PbSe, Materials Horizons 2(3) (2015) 323-329.

[51] T.M. Tritt, Thermal conductivity: theory, properties, and applications, Springer Science \& Business Media2005. 


\section{Figure Caption}

Figure 1. (a) XRD patterns and (b) enlarged XRD patterns from $36^{\circ}$ to $44^{\circ}$ for $\mathrm{Mg}_{1-\mathrm{x}} \mathrm{Ca}_{\mathrm{x}} \mathrm{Ag}_{0.97} \mathrm{Sb}_{0.99}$ samples ( $\mathrm{x}=0,0.0025,0.005$, and 0.01).

Figure 2. (a) Typical SEM image and (b) medium magnification TEM image for $\mathrm{Mg}_{0.995} \mathrm{Ca}_{0.005} \mathrm{Ag}_{0.97} \mathrm{Sb}_{0.99}$ sample.

Figure 3. High magnification TEM image for $\mathrm{Mg}_{0.995} \mathrm{Ca}_{0.005} \mathrm{Ag}_{0.97} \mathrm{Sb}_{0.99}$ sample. (a) Lamellar type-I (411) nanotwins and (b) spearhead shaped type-I (203) nanotwins; The inset corresponds the fast Fourier transformation (FFT) image along the $\mathrm{C}$ zone [011] axis direction.

Figure 4. Temperature dependent thermoelectric properties of $\mathrm{Mg}_{1-\mathrm{x}} \mathrm{Ca}_{\mathrm{x}} \mathrm{Ag}_{0.97} \mathrm{Sb}_{0.99}$ samples ( $\mathrm{x}=0,0.0025,0.005$ and 0.01). (a) electrical resistivity, (b) Seebeck coefficient, (c) power factor, (d) total thermal conductivity (e) lattice thermal conductivity, and (f) $Z T$.

Figure 5. Temperature dependent electrical transport properties for $\mathrm{MgAg}_{0.97} \mathrm{Sb}_{0.99-\mathrm{x}} \mathrm{M}_{\mathrm{x}}$ samples $(\mathrm{M}=\mathrm{As}, \mathrm{Bi}, \mathrm{Sn}$ and $\mathrm{Pb})(\mathrm{x}=0,0.005$ and 0.01). (a) Electrical resistivity, (b) Seebeck coefficient and (c) power factor for As and Bi doping. (d) Electrical resistivity, (e) Seebeck coefficient and (f) power factor for $\mathrm{Sn}$ and $\mathrm{Pb}$ doping.

Figure 6. The calculated partial DOS of $\alpha-\mathrm{MgAgSb}$ using DFT and GGA-PBE [31]. The vertical line denotes the Fermi energy.

Figure 7. Carrier concentration dependent electrical transport properties of $\mathrm{MgAgSb}$ based materials, including dopants $\mathrm{Li}$ [24], $\mathrm{Ca}, \mathrm{As}, \mathrm{Bi}, \mathrm{Sn}$ and $\mathrm{Pb}$. (a) Mobility, (b) electrical resistivity, (c) Seebeck coefficient, and (d) power factor. The dashed lines are calculated based on single parabolic band model with acoustic phonon scattering mechanism [44]. 

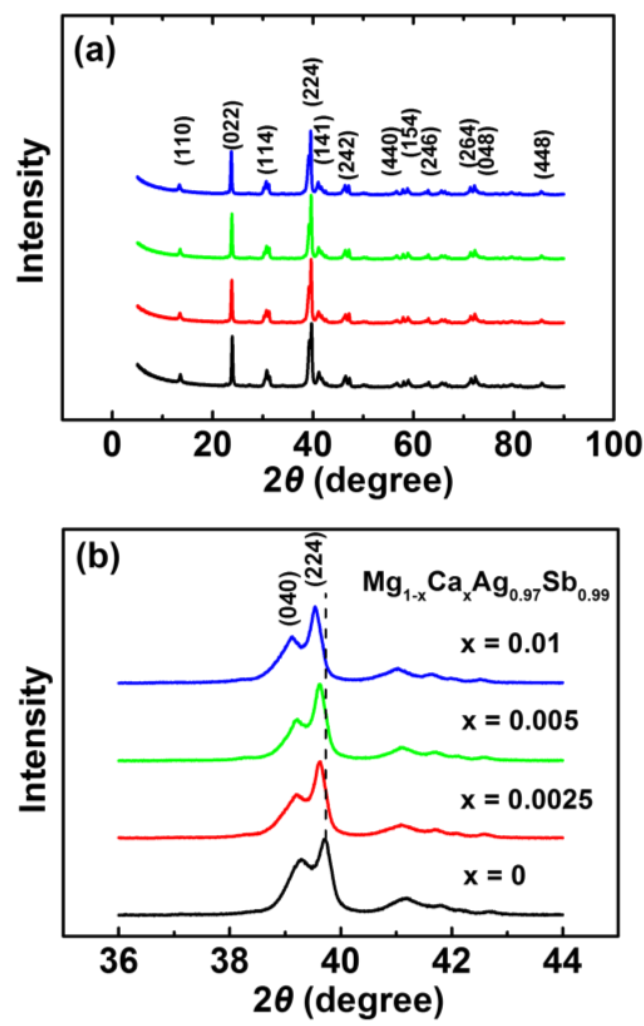

Figure 1 

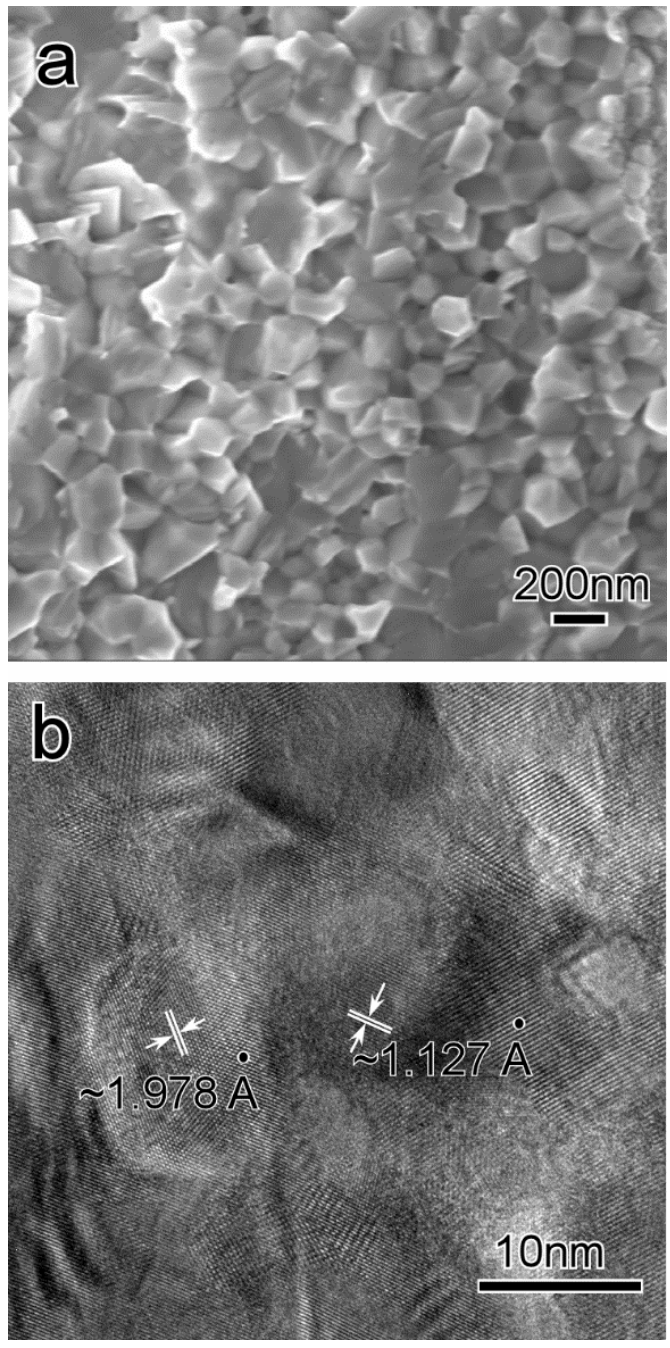

Figure 2 


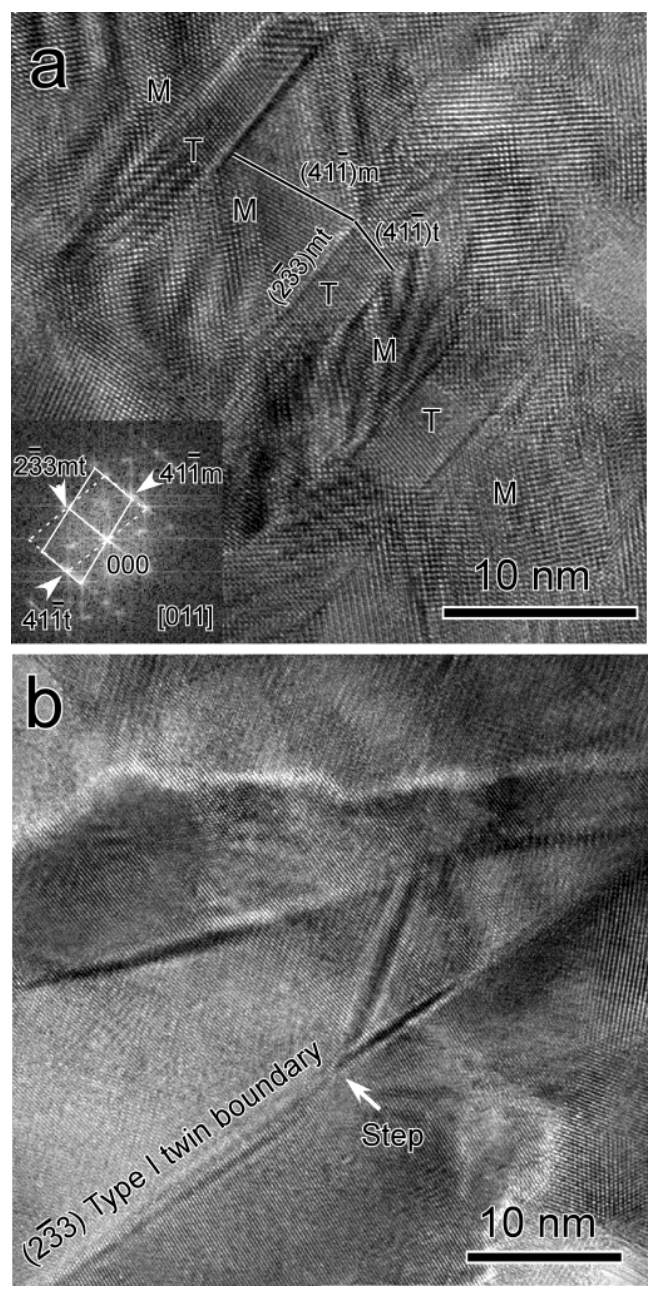

Figure 3 



Figure 4 



Figure 5 


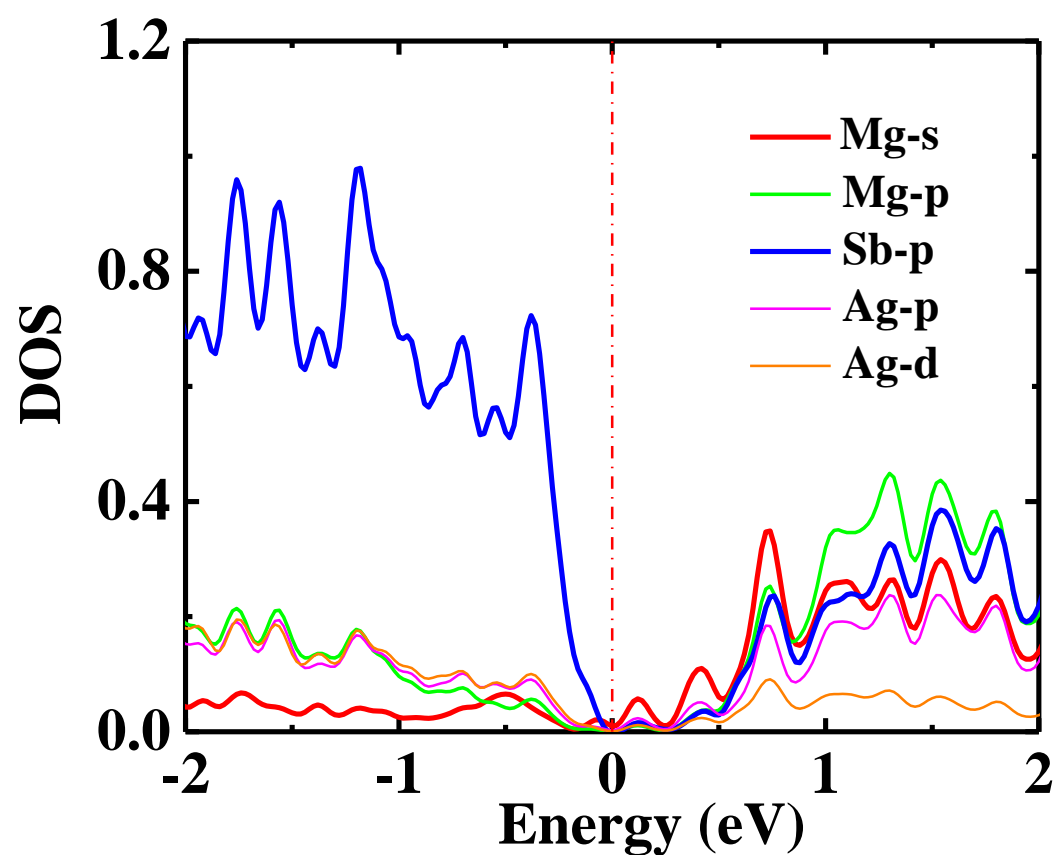

Figure 6 



Figure 7 
Table 1 Electrical transport properties for $\mathrm{Mg}_{1-\mathrm{x}} \mathrm{Ca}_{\mathrm{x}} \mathrm{Ag}_{0.97} \mathrm{Sb}_{0.99}$ samples ( $\mathrm{x}=0,0.0025$, 0.005 and 0.01 )

\begin{tabular}{ccccc}
\hline Composition & $n_{H}$ & $\mu_{H}$ & $m^{*}$ & $\eta$ \\
& $\left(10^{19} \mathrm{~cm}^{-3}\right)$ & $\left(\mathrm{cm}^{2} \mathrm{~V}^{-1} \mathrm{~s}^{-1}\right)$ & $\left(m_{e}\right)$ & \\
\hline $\mathrm{MgAg}_{0.97} \mathrm{Sb}_{0.99}$ & 2.7 & 64.9 & 2.35 & -0.75 \\
$\mathrm{Mg}_{0.9975} \mathrm{Ca}_{0.0025} \mathrm{Ag}_{0.97} \mathrm{Sb}_{0.99}$ & 3.1 & 64.3 & 2.38 & -0.61 \\
$\mathrm{Mg}_{0.995} \mathrm{Ca}_{0.005} \mathrm{Ag}_{0.97} \mathrm{Sb}_{0.99}$ & 4.1 & 63.2 & 2.39 & -0.18 \\
$\mathrm{Mg}_{0.99} \mathrm{Ca}_{0.01} \mathrm{Ag}_{0.97} \mathrm{Sb}_{0.99}$ & 5.7 & 60.8 & 2.38 & 0.09 \\
\hline
\end{tabular}


Table 2 Carrier concentration and mobility for $\mathrm{MgAg}_{0.97} \mathrm{Sb}_{0.99-\mathrm{x}} \mathrm{M}_{\mathrm{x}}$ samples (M=As, $\mathrm{Bi}, \mathrm{Sn}$ and $\mathrm{Pb})(\mathrm{x}=0.005$ and 0.01$)$

\begin{tabular}{ccc}
\hline Composition & $n_{H}\left(10^{19} \mathrm{~cm}^{-3}\right)$ & $\mu_{H}\left(\mathrm{~cm}^{2} \mathrm{~V}^{-1} \mathrm{~s}^{-1}\right)$ \\
\hline $\operatorname{MgAg}_{0.97} \mathrm{Sb}_{0.985} \mathrm{As}_{0.005}$ & 6.0 & 50.3 \\
$\operatorname{MgAg}_{0.97} \mathrm{Sb}_{0.98} \mathrm{As}_{0.01}$ & 8.1 & 45.1 \\
$\operatorname{MgAg}_{0.97} \mathrm{Sb}_{0.985} \mathrm{Bi}_{0.005}$ & 2.9 & 56.6 \\
$\operatorname{MgAg}_{0.97} \mathrm{Sb}_{0.98} \mathrm{Bi}_{0.01}$ & 3.1 & 49.6 \\
$\operatorname{MgAg}_{0.97} \mathrm{Sb}_{0.985} \mathrm{Sn}_{0.005}$ & 3.7 & 56.4 \\
$\operatorname{MgAg}_{0.97} \mathrm{Sb}_{0.985} \mathrm{Sn}_{0.01}$ & 5.0 & 48.4 \\
$\operatorname{MgAg}_{0.97} \mathrm{Sb}_{0.985} \mathrm{~Pb}_{0.005}$ & 3.2 & 55.6 \\
$\operatorname{MgAg}_{0.97} \mathrm{Sb}_{0.985} \mathrm{~Pb}_{0.01}$ & 3.5 & 52.9 \\
\hline
\end{tabular}



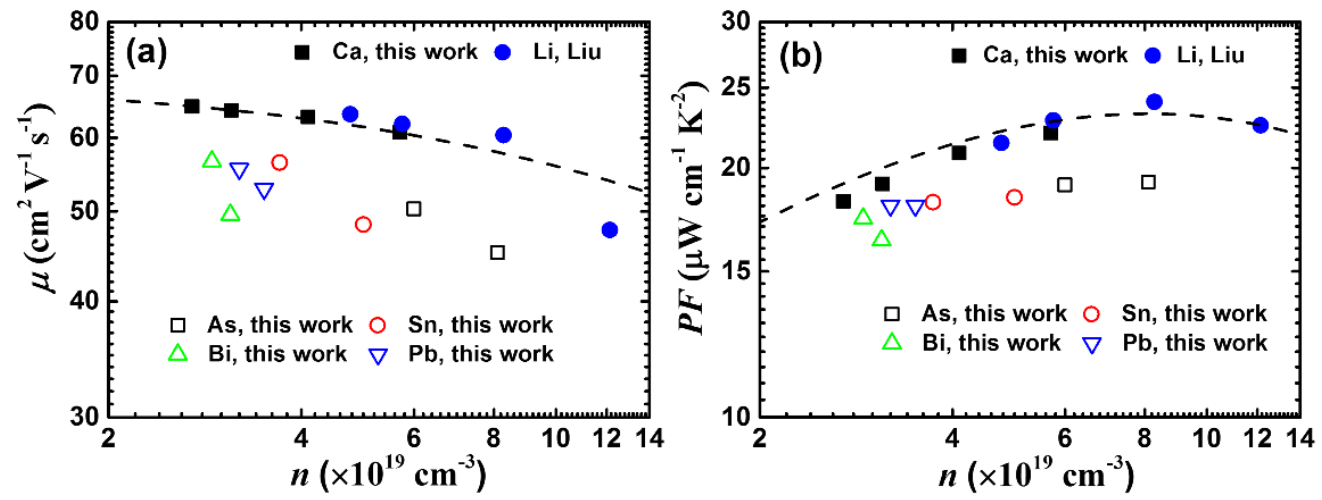